\title{
Informed feature regularization in voxelwise modeling for naturalistic fMRI experiments
}

\author{
Özgür Yılmaz ${ }^{1,2}$ (D) $\mid$ Emin Çelik $^{1,3} \mid$ Tolga Çukur ${ }^{1,2,3}$ (i)
}

\author{
${ }^{1}$ National Magnetic Resonance Research \\ Center, Bilkent University, Ankara, Turkey \\ ${ }^{2}$ Department of Electrical and Electronics \\ Engineering, Bilkent University, Ankara, \\ Turkey \\ ${ }^{3}$ Neuroscience Program, Sabuncu Brain \\ Research Center, Bilkent University, \\ Ankara, Turkey

\section{Correspondence} \\ Tolga Çukur, Department of Electrical and \\ Electronics Engineering, Bilkent University, \\ Ankara TR-06800, Turkey. \\ Email: cukur@ee.bilkent.edu.tr

\section{Funding information} \\ National Eye Institute Grant, Grant/Award \\ Number: EY019684; Marie Curie Action \\ Career Integration Grant, Grant/Award \\ Number: PCIG13-GA-2013-618101; \\ European Molecular Biology Organization \\ Installation Grant, Grant/Award Number: \\ IG 3028; TUBA GEBIP 2015 fellowship; \\ BAGEP 2017 fellowship; ASELSAN PhD \\ scholarship; TUBITAK-BIDEB 2211 \\ scholarship; NVIDIA GPU Grant
}

\begin{abstract}
Voxelwise modeling is a powerful framework to predict single-voxel functional selectivity for the stimulus features that exist in complex natural stimuli. Yet, because VM disregards potential correlations across stimulus features or neighboring voxels, it may yield suboptimal sensitivity in measuring functional selectivity in the presence of high levels of measurement noise. Here, we introduce a novel voxelwise modeling approach that simultaneously utilizes stimulus correlations in model features and response correlations among voxel neighborhoods. The proposed method performs feature and spatial regularization while still generating single-voxel response predictions. We demonstrated the performance of our approach on a functional magnetic resonance imaging dataset from a natural vision experiment. Compared to VM, the proposed method yields clear improvements in prediction performance, together with increased feature coherence and spatial coherence of voxelwise models. Overall, the proposed method can offer improved sensitivity in modeling of single voxels in naturalistic functional magnetic resonance imaging experiments.
\end{abstract}

\section{KE Y W O R D S}

computational neuroscience, feature regularization, modeling, stimulus correlation

\section{1 | INTRODUCTION}

Voxelwise modeling (VM) is a powerful framework to model single-voxel functional selectivity for the multitude of stimulus features that exist in natural stimuli (Kay, Naselaris, Prenger, \& Gallant, 2008; Naselaris, Prenger, Kay, Oliver, \& Gallant, 2009). Previous studies have used this approach to sensitively model a broad range of representations from low-level spatiotemporal features to high-level object and action categories (Çukur, Nishimoto, Huth, \& Gallant, 2013; Huth, Nishimoto, Vu, \& Gallant, 2012; Lescroart, Stansbury, \& Gallant, 2015; Nishimoto et al., 2011). In VM, an encoding model is constructed that contains stimulus features hypothesized to

\footnotetext{
Abbreviations: BOLD, blood-oxygen-level-dependent; FI-VM, feature-informed voxelwise modeling; FMRI, functional magnetic resonance imaging; JI-VM, jointly informed voxelwise modeling; ROI, region of interest; SI-VM, spatially informed voxelwise modeling; VM, voxelwise modeling.
} 
elicit blood-oxygen-level-dependent (BOLD) responses in cortical voxels. For each voxel, a weighted linear combination of model features that best explain measured BOLD responses is computed via regularized regression. To avoid over-fitting and ensure generalizability to new data, it is common to perform L2-norm regularization over model features with a uniform prior to penalize large model weights (Bishop, 2013). The regularization parameter is separately selected for each voxel based on a cross-validation procedure. Following parameter selection, the resulting model weights characterize the functional selectivity of single voxels.

Classical VM performs independent modeling to maximize sensitivity to single voxels, yet it disregards potential response correlations among neighboring voxels across cortex (Erwin, Obermayer, \& Schulten, 1995; Zarahn, Aguirre, \& D'Esposito, 1997) as well as potential stimulus correlations among model features (Nunez-Elizalde, Huth, \& Gallant, 2018). This in turn might reduce the sensitivity of VM in capturing functional selectivity in the presence of high levels of measurement noise. To better utilize response correlations, we recently proposed a spatial regularization term for VM via a graph Laplacian approach where neighborhoods were defined in volumetric brain space (Çelik, Dar, Yılmaz, Keleş, \& Çukur, 2019). Spatially regularized VM models were found to improve model performance broadly across cerebral cortex. Yet, the proposed approach still uses L2-norm regularization over model features-a uniform Gaussian prior-that reflects the assumption that model features are uncorrelated (Hoerl \& Kennard, 1970). This assumption might be suboptimal for models of natural stimuli. For example, in a category model that contains distinct object and action categories within natural scenes, the stimulus time courses for similar categories are correlated (e.g., "human"-"hand," "kid"-"run," "building"-“road" and "park""tree"; Huth et al., 2012). Moreover, a voxel selective for humans will typically yield elevated responses to body parts, animate categories or human-related tools compared to unrelated categories (Huth et al., 2012). As voxelwise functional selectivity profiles for related categories are expected to be similar, model weights are not truly uncorrelated (NunezElizalde et al., 2018).

In this study, we propose a new VM approach to increase sensitivity in modeling functional selectivity (Figure 1). First, we introduce an informed feature regularization that takes into account correlations among model features. This is accomplished by enforcing similarity of model weights among features that are correlated to each other. To further boost model performance, we introduce a new spatial regularization term. We had previously proposed to define voxel neighborhoods based on Euclidean distances in volumetric brain space (Çelik et al., 2019). Yet, distances along the cortical surface are more likely to capture functional similarities among neighboring voxels (Tucholka, Fritsch, Poline, \& Thirion, 2012; Van Essen, Drury, Joshi, \& Miller, 1998). Therefore, here we select voxel neighborhoods using geodesic distances on the cortical surface. To achieve this goal, we measured intervoxel distances using inflated cortical spaces (Gao, Huth, Lescroart, \& Gallant, 2015).

To independently evaluate improvements enabled by informed feature regularization and spatial regularization, we implemented three variants of VM together with Classical VM: a method that only uses informed feature regularization (feature-informed voxelwise modeling, FI-VM), a method that only uses spatial regularization (spatially informed voxelwise modeling, SI-VM) and a method that simultaneously uses feature and spatial regularizations (jointly informed voxelwise modeling, JI-VM). Demonstrations were performed on a category model fit to whole-brain BOLD responses recorded while subjects watched natural movies. To measure correlations among 1,705 object and action category features in the model, we evaluated the pairwise similarities of the categories in a word embedding space obtained from a large text corpus. These similarities were then input to a graph Laplacian term, to form the feature and spatial regularization terms. The VM variants were compared in terms of their prediction scores and spatial and feature coherence of the resulting model weights.

\section{2 | MATERIALS AND METHODS}

\section{1 | MRI protocols}

Magnetic resonance imaging (MRI) data were collected on a 3T Siemens Tim Trio scanner at the University of California, Berkeley, using a 32-channel Siemens volume coil. Functional scans were collected using a gradient EPI sequence with repetition time $=2.00 \mathrm{~s}$, echo time $=31 \mathrm{~ms}$, flip angle $=70^{\circ}$, voxel size $=2.24 \times 2.24 \times 4.1 \mathrm{~mm}^{3}$, slice thickness $=3.5 \mathrm{~mm}$ with $18 \%$ slice gap, matrix size $100 \times 100$, and field of view $=224 \times 224 \mathrm{~mm}^{2}$ and 32 axial slices.

Anatomical data for three subjects were collected using a $\mathrm{T}_{1}$-weighted multi-echo MP-RAGE sequence on the same 3T scanner. Anatomical data for the other two subjects were collected on a 1.5T Philips Eclipse scanner.

\subsection{Subjects}

Functional data were collected from five healthy male subjects: S1 (age 25), S2 (age 25), S3 (age 25), S4 (age 32 ) and S5 (age 29). All subjects had normal or corrected-to-normal vision. The experimental protocol was approved by the Committee for the Protection of Human Subjects at the University of California, Berkeley. Prior 
Natural Movies

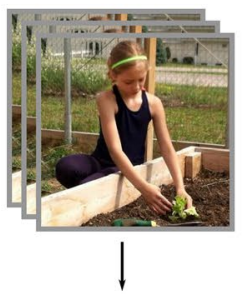

(a)
Stimulus Labeling
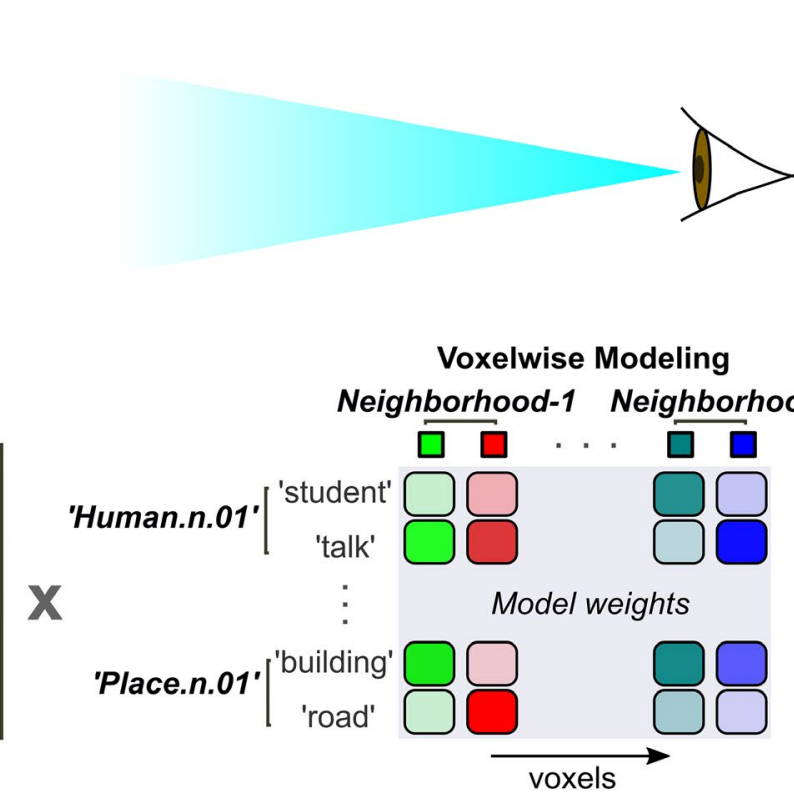

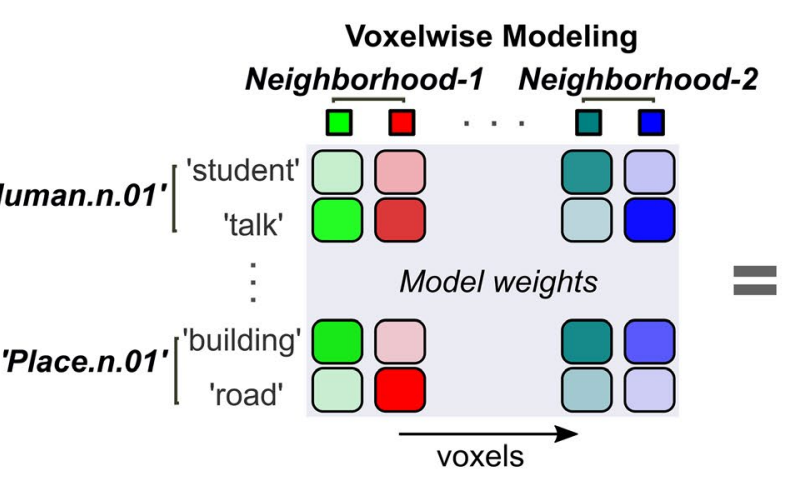

fMRI Scan

Neighborhood-1

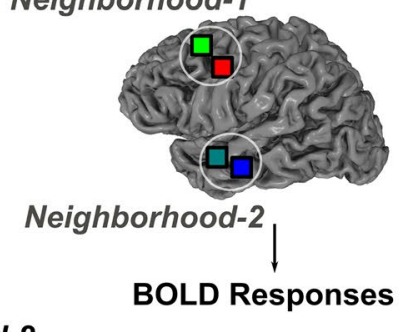

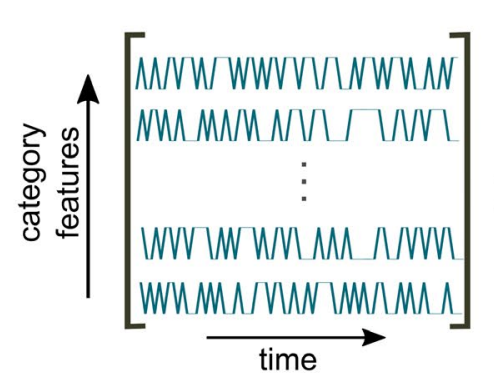
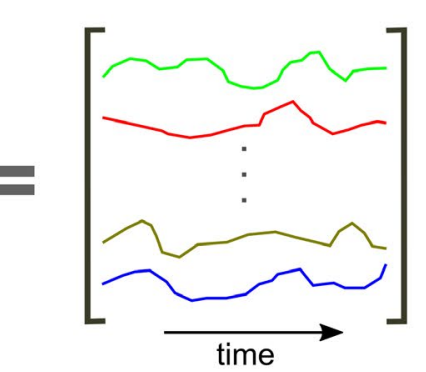

(b) Neighborhood-1 Neighborhood-2 Neighborhood-1 Neighborhood-2 Neighborhood-1 Neighborhood-2
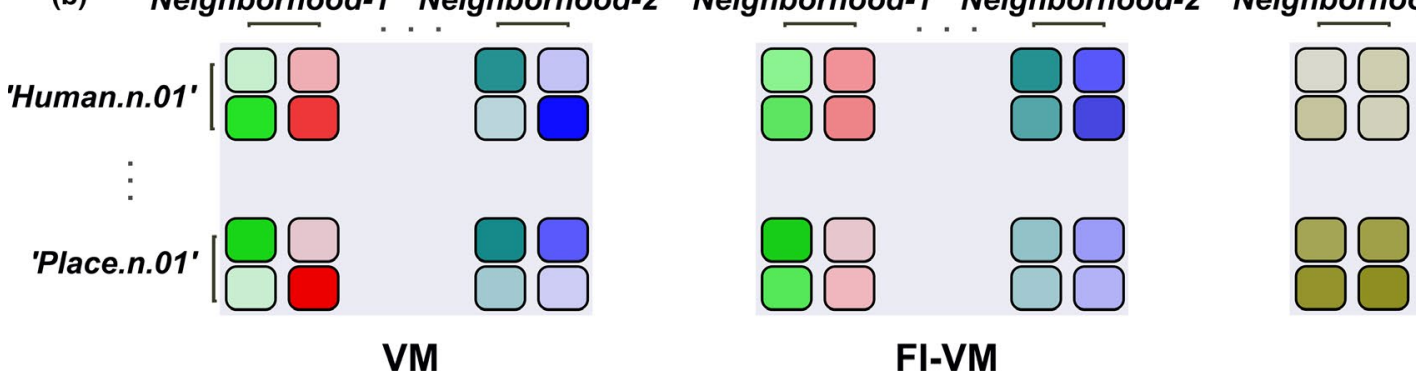

FI-VM

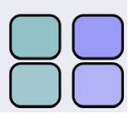

\section{JI-VM}

F I G U RE 1 Natural movie experiment and model fitting. (a) Whole-brain BOLD responses were acquired while subjects viewed natural movies. To estimate functional selectivity in single voxels, we fit voxelwise models that optimally predict measured BOLD responses in terms of the category features present in the movie stimuli (Huth et al., 2012). The resulting models describe how each of the 1,705 object and action categories in the movie stimulus evokes BOLD responses. (b) Classical voxelwise modeling (VM) ignores potential correlations in stimulus features and correlations among neighboring voxels. Feature-informed voxelwise modeling (FI-VM) takes into account correlations among model features to increase accuracy in predicting single-voxel selectivity. The resulting models have increased feature coherence. Jointly informed voxelwise modeling (JI-VM) further incorporates shared information between neighboring voxels. The resulting voxelwise models have both increased feature and spatial coherence [Colour figure can be viewed at wileyonlinelibrary.com]

to scanning, written informed consent was obtained from all subjects.

\section{3 | Experimental procedure}

The movie stimulus was identical to the set used in Nishimoto et al. (2011). The stimulus contained short clips (10-20 s) of color natural movies taken either from Apple QuickTime gallery (http://trailers.apple.com/) or from YouTube (http:// www.youtube.com/). The list of selected movies is as follows: "Artbeats HD," "Australia," "Bolt," "BBC Motion Gallery," "Bride Wars," "Changeling," "Duplicity," "Fuel," "Hotel for Dogs," "IGN Game of the Year 2008," "Ink Heart," "JAL Boeing 747 landing Kai Tak," "King Lines," "Madagascar 2," "Mall Cop," "Mammoth HD," "Pink Panther 2," "Proud American," "Role Models," "Shark Water," "Star Trek," "The
American Recovery and Reinvestment Plan," "The Macaulay Library," "The Tale of Despereaux," "Warren Miller Higher Ground," "Where the hell is Matt?" and "Yes Man." Original movie frames were cropped to square form and downsampled to $512 \times 512$ pixels. The stimulus was presented on $24^{\circ} \times 24^{\circ}$ degrees of visual angle. A fixation spot $\left(0.16^{\circ}\right.$ square $)$ was superimposed on the center of the screen and its color was alternated at $3 \mathrm{~Hz}$, to make it continuously visible. For model estimation, 7,200 s (3,600 time samples, TR = $2 \mathrm{~s})$ of movies were presented to the subjects in 12 separate 10-min runs. For model validation, a different collection of 9 separate 1-min movie clips were selected. The selected clips were aggregated in randomized order to create in 9 separate 10-min runs. As such, each 1-min validation clip was presented to the subjects 10 times, and functional magnetic resonance imaging (fMRI) data were averaged across the 10 repeats to alleviate noise. These procedures resulted in 270 time samples of validation 
data. To minimize the effects of hemodynamic transients, data obtained in the first $10 \mathrm{~s}$ of each run were omitted. Note that the same data were analyzed in several previous studies (Çelik et al., 2019; Çukur, Huth, Nishimoto, \& Gallant, 2013; Çukur, Nishimoto, et al., 2013; Huth et al., 2012).

\section{4 | ROI abbreviations}

Regions of interest (ROIs) used in our study are as follows: visual areas (V1-V4, V7), fusiform face area (FFA), extrastriate body area (EBA), occipital face area (OFA), parahippocampal place area (PPA), occipital place area (OPA), retrosplenial cortex (RSC), intraparietal sulcus (IPS), lateral occipital visual area (LO), frontal eye fields (FEF), supplementary eye fields (SEF), frontal operculum (FO), primary motor and somatosensory foot areas (M1F, S1F), primary motor and somatosensory hand areas $(\mathrm{M} 1 \mathrm{H}, \mathrm{S} 1 \mathrm{H})$, primary motor and somatosensory mouth areas (M1M, S1M), supplementary motor hand area (SMHA) and supplementary motor foot area (SMFA).

\section{5 | Functional localizers}

For each subject, we defined boundaries of common functional ROIs by using three sets of functional localizers: a visual category localizer, a retinotopic localizer and a motor localizer.

In the visual category localizer, each subject was shown colored images of either places, faces, human body parts, animals, household objects or spatially scrambled household objects. The localization data were collected in 64.5 -min runs. A single run consisted of 16 blocks, each $16 \mathrm{~s}$ long and separated with 500-ms interblock intervals. In each block, 20 images of either places, faces, human body parts, animals, household objects or spatially scrambled household objects were displayed. Each image was shown for $300 \mathrm{~ms}$ after a 500-ms interstimulus interval. Fusiform face area (FFA) and occipital face area (OFA) were defined using the faces versus objects contrast (Kanwisher, McDermott, \& Chun, 1997). Extrastriate body area (EBA) was defined using the human body parts versus objects contrast (Downing, Jiang, Shuman, $\&$ Kanwisher, 2001). Parahippocampal place area (PPA), retrosplenial cortex (RSC) and occipital place area (OPA) were defined using the scenes versus objects contrast (Epstein \& Kanwisher, 1998; Nakamura, 2000).

Retinotopic mapping data were collected in four 9-min scans. The subjects were shown clockwise and counterclockwise rotating wedges in two scans, expanding and contracting rings in the remaining two scans. V1, V2, V3, V4, V7 and LO were defined based on visual angle and eccentricity maps. Using V1, V2 and V3, a broad retinotopic area (RET) was defined.

Motor localizer data were collected in a single 10-min scan. Each subject performed 6 separate motor tasks in random blocks of $20 \mathrm{~s}$. During the "hand" cue, the subject made small finger-drumming movements with both hands. During the "foot" cue, the subject made small toe movements. During the "mouth" cue, the subject made small mouth movements mimicking the syllable balabalabala. During the "speak" cue, the subject subvocalized self-generated sentences. During the "saccade" cue, the subject performed rapid saccades between different targets on the screen. During the "rest" cue, the subject did not perform any motor tasks. Contrast between the "saccade" and "rest" conditions was used to define intraparietal sulcus (IPS), frontal operculum (FO), frontal eye fields (FEF) and supplementary eye fields (SEF). Contrast between "foot" and "rest" was used to define primary motor and somatosensory foot areas (M1F, S1F) and supplementary motor foot area (SMFA). Contrasts between "hand" and "rest" were used to define primary motor and somatosensory hand areas $(\mathrm{M} 1 \mathrm{H}$, $\mathrm{S} 1 \mathrm{H})$ and supplementary motor hand area (SMHA). Contrast between "mouth" and "rest" was used to define primary motor and somatosensory mouth areas (M1M, S1M). In this study, we aimed to examine how object and action features in natural movies modulate BOLD responses in single voxels. As it is commonly considered that motor and somatosensory areas do not play a major role in visual category representation, voxels within these regions were excluded from subsequent analyses.

\section{6 | Data preprocessing}

Motion correction and image realignment were done using the Motion Correction FMRIB Linear Image Registration Tool (MCFLIRT) in FSL (Woolrich et al., 2009). Functional images of each subject were registered to a preselected image of that subject. The final time series data were manually checked for accuracy. The resulting time courses were $z$ scored individually for each voxel. No spatial smoothing was applied to the fMRI data.

Detrending was used to remove low-frequency drifts in BOLD responses. The drifts were estimated using a median filter with a 120-s window and then subtracted from measured BOLD responses.

Brain Extraction Tool in FSL was used to eliminate non-cortical voxels. The set of voxels within a $4 \mathrm{~mm}$ radius of the cortical surface were defined as cortical voxels. Following analyses were done on a total of $35,158 \pm 887$ cortical voxels (mean $\pm S D$ across subjects).

\subsection{Cortical flat maps}

Cortical flat maps of subjects were generated using FreeSurfer (Dale, Fischl, \& Sereno, 1999). These flat maps are based on $\mathrm{T}_{1}$-weighted anatomical scans. Anatomical surface segmentations were manually checked and corrected 
using Pycortex (Gao et al., 2015). Relaxation cuts were made into the surface of each hemisphere, and the surface crossing the corpus callosum was removed. The calcarine sulcus cut was made at the horizontal meridian in V1. Functional images were aligned to the cortical flat maps using boundarybased registration (BBR) in FSL. For visualization of flat maps, the mid-cortical surface that lies halfway between the pial and white matter surfaces was selected.

\section{8 | Category model}

To extract category information present in the natural movie stimulus, we used a category model (Çukur, Nishimoto, et al., 2013; Huth et al., 2012). We tagged object and action categories in each 1-s portion of the natural movies using the WordNet lexicon (Miller \& Miller, 1995). Finally, a list of features including 1,705 distinct object and action categories was formed. Note that, the same list of object and action categories (1,705 features in total) was used in all four modeling methods. The time courses of the features were resampled using a 3-lobe Lanczos filter with a cutoff frequency of $0.249 \mathrm{~Hz}$ (the Nyquist frequency of the fMRI acquisition). To reduce dimensionality and improve model fits, we applied PCA onto the resulting stimulus matrix. We selected only the first 300 PCs that explain $89.2 \%$ of the variance in the stimulus. To rule out possible biases due to correlations between category model and global motion energy, we added a nuisance regressor that reflects the total motion energy in the movie stimulus. To obtain the total motion energy, we summed the output of all spatiotemporal Gabor filters used in a separate motion energy model (Çelik et al., 2019).

\section{9 | Voxelwise model estimation and validation}

We used a voxelwise modeling framework to fit between stimulus and BOLD responses (Çukur, Nishimoto, et al., 2013; Huth et al., 2012). In the Classical VM approach, $\mathrm{L}_{2}-$ regularized linear regression is used to predict how each feature modulates measured BOLD responses. A category model was fit to measure voxelwise selectivity for high-level object and action categories (Huth et al., 2012). To account for hemodynamic delays, a finite-impulse-response (FIR) filter with three time delays $(4,6$ and $8 \mathrm{~s})$ was applied to each model feature before fitting the model.

$$
X \times W=Y
$$

where $Y$ is the response matrix of size (number of TRs $\times$ number of voxels), $X$ is the stimulus matrix of size (number of
TRs $\times(3 \times$ number of features $))$, and $W$ is the weight matrix of size $((3 \times$ number of features $) \times$ number of voxels $)$. In $\mathrm{VM}, \mathrm{L}_{2^{-}}$ regularized regression is used to minimize the cost function:

$$
\min _{w_{i}} \sum_{i}\left\|X w_{i}-y_{i}\right\|_{2}^{2}+\lambda \sum_{i=1,2, \ldots, N_{\mathrm{vox}}}\left\|w_{i}\right\|_{2}^{2}
$$

where $w_{i}$ is the vector containing category model weights for voxel- $i$, and $y_{i}$ is the vector containing the time course of BOLD responses of voxel- $i$. Cost function can be expressed in matrix form as follows:

$$
\begin{gathered}
\min _{w_{i}} \operatorname{Tr}\left[(X W-Y)^{T}(X W-Y)\right]+\lambda \operatorname{Tr}\left(W^{T} W\right) \\
\min _{w_{i}} \operatorname{Tr}\left(W^{T} X^{T} X W\right)-2 \operatorname{Tr}\left(Y^{T} X W\right)+\operatorname{Tr}\left(Y^{T} Y\right)+\lambda \operatorname{Tr}\left(W^{T}\right. \\
i=1,2, \ldots, N_{\mathrm{vox}}
\end{gathered}
$$

Data from single voxels were aggregated into matrix form to speed up computations. Note that the minimization was still performed for each voxel separately as each column of the weight matrix $(W)$ is the vector containing category model weights for the corresponding voxel and the minimization procedure does not involve any interaction among the columns. In Equation 3, by setting the gradient with respect to $W$ to zero, we have:

$$
\begin{aligned}
& 2 X^{T} X W-2 X^{T} Y+2 \lambda W=0 \\
& X^{T} X W+\lambda W=X^{T} Y \\
& \left(X^{T} X+\lambda I\right) W=X^{T} Y \\
& W=\left(X^{T} X+\lambda I\right)^{\dagger} X^{T} Y
\end{aligned}
$$

To obtain model weights and to measure prediction scores, a 100-fold cross-validation was used. In each fold, a total of 3,600 samples were split into a contiguous block of 3,000 samples for model fitting and a remaining contiguous block of 600 test samples for selection of $\mathrm{L}_{2}$ regularization parameter $(\lambda)$. Separate models were fit for 30 different regularization parameters in the range of $10^{-2}$ to $10^{7}$ (spaced logarithmically). We calculated prediction scores based on the coefficient of determination (i.e., explained variance, $R^{2}$ ) between measured and predicted BOLD responses. Separately for each voxel, we picked the regularization parameter that maximized the average prediction score across folds. Final voxelwise models based on the optimal parameters were refit using the entire 3,600 time samples.

To measure final prediction scores, a 1,000-fold jackknife resampling at a rate of $80 \%$ was used on a separate validation data (270 samples). Separately for each voxel, final prediction score was taken as the average prediction score across jackknife folds. For assessment of model performance, we then computed the average prediction score across five subjects in each functional ROI. 


\subsection{0 | Parameter optimization}

In the proposed approach, there are five hyperparameters to be optimized. Three of these parameters $\left(\lambda, \lambda_{f}\right.$ and $\left.\lambda_{s}\right)$ are set separately for individual voxels in each method. The remaining two hyperparameters (the standard deviation of Gaussian filter and the feature similarity threshold) determine the feature Laplacian matrix $(F)$. The feature Laplacian matrix $F$ is of size $N_{\text {feat }} \times N_{\text {feat }}$, where $N_{\text {feat }}$ is the number of category features. $F=T-S$, where $s_{j k}$ (entries of matrix $S$, see Equation 5) is the relatedness metric of the feature- $j$ and feature- $k$ (high for related features, and low or zero for distant features), and $T$ is a diagonal matrix with $T_{j j}=\sum_{k} s_{j k}$

$$
s_{j k}=\left\{\begin{array}{c}
\exp \left(\frac{-\left(1-c s_{j k}\right)^{2}}{2 \sigma^{2}}\right), \quad c s_{j k} \geq d_{\text {sim }} \\
0, \quad c s_{j k}<d_{\text {sim }}
\end{array}\right.
$$

$s_{j k}$ is calculated within a word embedding space (Deerwester, Dumais, Furnas, Landauer, \& Harshman, 1990; Lund \& Burgess, 1996; Mitchell et al., 2008; Turney \& Pantel, 2010; Wehbe et al., 2014). The word embedding space was formed based on word co-occurrence statistics. The assumption here is that words with similar meaning tend to occur in nearby positions in text. The co-occurrence statistics were taken from a large corpus of text. This corpus contained words from transcripts of many popular books, Wikipedia pages and user comments gathered from reddit. com (604 popular books, 2,405,569 Wikipedia pages and $36,333,459$ user comments scraped from reddit.com). In the corpus, 10,470 distinct words appeared 1,548,774,960 times (Huth, de Heer, Griffiths, Theunissen, \& Gallant, 2016). Cooccurrences among the words from a lexicon of 10,470 words and the 985 basis words (from Wikipedia's List of 1,000 Basic Words) were calculated. The appearance of a lexicon word within a 15-word neighborhood of the basis word was taken as a single co-occurrence. We reduced the word embedding space by projecting onto the PC space obtained in Category Model. As a result, each of the 1,705 category features had a 300-dimensional vector representing its location in the reduced word embedding space. We then computed the similarity (cosine similarity, $c s_{j k}$ ) of each feature pair. We eliminated feature pairs that have a similarity smaller than the feature similarity threshold $\left(d_{\text {sim }}\right)$. Later, we fed those similarity values into a Gaussian filter, to get the final form of the relatedness metric $\left(s_{j k}\right)$ as in Equation 5.

Standard deviation $(\sigma)$ and feature similarity threshold $\left(d_{\text {sim }}\right)$ were optimized a priori via a cross-validation procedure on the training data. To do this, we computed voxelwise prediction scores of separate JI-VM models with standard deviations of $[0.1,0.2,0.4,0.8,1.2,2.0]$ and feature similarity thresholds of $[0.1,0.2,0.3,0.5]$. The model with a standard deviation $(\sigma)$ of 0.4 and a feature similarity threshold $\left(d_{\text {sim }}\right)$ of 0.2 outperformed others in most ROIs; thus, we fixed these parameters throughout our study.

To incorporate spatial regularization into VM, we constructed a spatial Laplacian matrix that represents correlated information across neighboring voxels. The spatial Laplacian matrix $(L)$ is of size $N_{\text {vox }} \times N_{\text {vox }}$, where $N_{\text {vox }}$ is the number voxels in the subject. $L=P-C$, where $c_{i l}$ (entries of matrix $C$ ) corresponds to the proximity of voxel- $i$ and voxel- $l$ in three-dimensional space (high for immediate neighbors, and low or zero for voxels far away), and $P$ is a diagonal matrix with $P_{i i}=\sum_{l} c_{i l}$. The weight $c_{i l}$ represents the cost of having weights of voxel- $i$ and voxel- $l$ more dissimilar to each other. To compute $c_{i l}$, first the distance between voxel- $i$ and voxel- $l$ is calculated as the Euclidean distance between those voxels in inflated cortical space (Gao et al., 2015). Calculated distances are then input to a Gaussian filter. We have two hyperparameters in constructing the spatial Laplacian matrix $L$ : the proximity limit after which two voxels are treated as spatially uncorrelated and the standard deviation of the Gaussian filter. Here, we prescribed these hyperparameters reported by Çelik et al. (2019) by converting into millimeter scale to use in our approach.

The other three hyperparameters are regularization parameters of $\mathrm{L}_{2}$ regularization, feature regularization and spatial regularization: $\lambda, \lambda_{f}$ and $\lambda_{s}$, respectively. $\lambda$ indicates the degree of $\mathrm{L}_{2}$ regularization across model features. $\lambda_{f}$ indicates the degree of penalization applied to the differences within the weights of related feature pairs. $\lambda_{s}$ indicates the degree of spatial regularization across category features of neighboring voxels. As voxels across cortex may need varying degrees of regularization, we optimized these parameters separately for each voxel.

\subsection{1 | JI-VM model estimation and validation}

Cost function for the regularized regression in the proposed approach can be expressed as follows:

$$
\begin{aligned}
& \min _{w_{i}} \sum_{i}\left\|X w_{i}-y_{i}\right\|_{2}^{2}+\lambda \sum_{i}\left\|w_{i}\right\|_{2}^{2}+\lambda_{f} \sum_{i} \sum_{j, k} s_{j k}\left(w_{i j}-w_{i k}\right)^{2} \\
& +\lambda_{s} \sum_{i, l} c_{i l}\left\|w_{i}-w_{l}\right\|_{2}^{2}
\end{aligned}
$$

where the third term is the feature regularization term, and the last term is the spatial regularization term. In the feature regularization term, the weighted sum of Euclidean distances between each feature pair is calculated for each voxel separately. $s_{j k}$ represents the cost of having model weights for feature- $j$ and feature- $k$ dissimilar to each other (Equation 5). In the spatial regularization term, the weighted sum of Euclidean distances between weights of voxels is calculated. The weight $c_{i l}$ represents the cost of having vectors containing category 
model weights of voxel- $i$ and voxel- $l$ dissimilar to each other (see Section 2.10).

$$
\left(X^{T} X+\lambda I\right) W+\lambda_{s} W L=X^{T} Y
$$

Cost function can be expressed in matrix form as follows:

$$
\begin{gathered}
\min _{w_{i}} \operatorname{Tr}\left[(X W-Y)^{T}(X W-Y)\right]+\lambda \operatorname{Tr}\left(W^{T} W\right)+\lambda_{f} \operatorname{Tr}\left(W^{T} F W\right)+\lambda_{s} \operatorname{Tr}\left(W L W^{T}\right) \\
\min _{w_{i}} \operatorname{Tr}\left(W^{T} X^{T} X W\right)-2 \operatorname{Tr}\left(Y^{T} X W\right)+\operatorname{Tr}\left(Y^{T} Y\right)+\lambda \operatorname{Tr}\left(W^{T} W\right)+\lambda_{f} \operatorname{Tr}\left(W^{T} F W\right)+\lambda_{s} \operatorname{Tr}\left(W L W^{T}\right) \\
\text { for } i=1,2, \ldots, N_{\mathrm{vox}}
\end{gathered}
$$

Then, by setting the gradient with respect to weight matrix $(W)$ to zero, we now have:

$$
\begin{aligned}
& 2 X^{T} X W-2 X^{T} Y+2 \lambda W+2 \lambda_{f} F^{T} W+2 \lambda_{s} W L=0 \\
& X^{T} X W+\lambda W+\lambda_{f} F^{T} W+\lambda_{s} W L=X^{T} Y \\
& \left(X^{T} X+\lambda I+\lambda_{f} F^{T}\right) W+\lambda_{s} W L=X^{T} Y
\end{aligned}
$$

To simplify Equation 8, we define $\mathrm{K}=X^{T} X, M=X^{T} Y$ and $B=\lambda_{s} L$ :

$$
\begin{aligned}
& \left(K+\lambda I+\lambda_{f} F^{T}\right) W+W B=M \\
& A W+W B=M
\end{aligned}
$$

\subsection{2 | Pseudo-code for JI-VM implementation}

Equation 9 cannot be solved with a single pseudo-inverse. An efficient algorithm for the solution from Çelik et al. (2019) is given in Table 1.

\subsection{3 | FI-VM and SI-VM model estimation and validation}

In FI-VM, we only include feature regularization term; therefore, $\lambda_{s}$ is set to 0 in Equation 8:

$$
\left(X^{T} X+\lambda I+\lambda_{f} F^{T}\right) W=X^{T} Y
$$

Solution to this equation is as follows:

$$
W=\left(X^{T} X+\lambda I+\lambda_{f} F^{T}\right)^{\dagger} X^{T} Y
$$

As the solution can be expressed via a single pseudo-inverse, we implemented the same cross-validation procedure as in Classical VM. This time, separate voxelwise models were fit for $30 \times 30$ different regularization parameter pairs $\left(\lambda, \lambda_{f}\right)$, each in the range from $10^{-2}$ to $10^{7}$ (spaced logarithmically). We measured the prediction scores using the same jackknife resampling method.

In SI-VM modeling, on the other hand, we removed the feature regularization term from JI-VM, equivalently setting $\lambda_{f}=0$ in Equation 8:
The implementation for SI-VM is the same as JI-VM case with setting $A=(K+\lambda I)$ and omitting the second loop with $\lambda_{f}$ from pseudo-code (see Section 2.12).

T A B L E 1 Pseudo-code for JI-VM

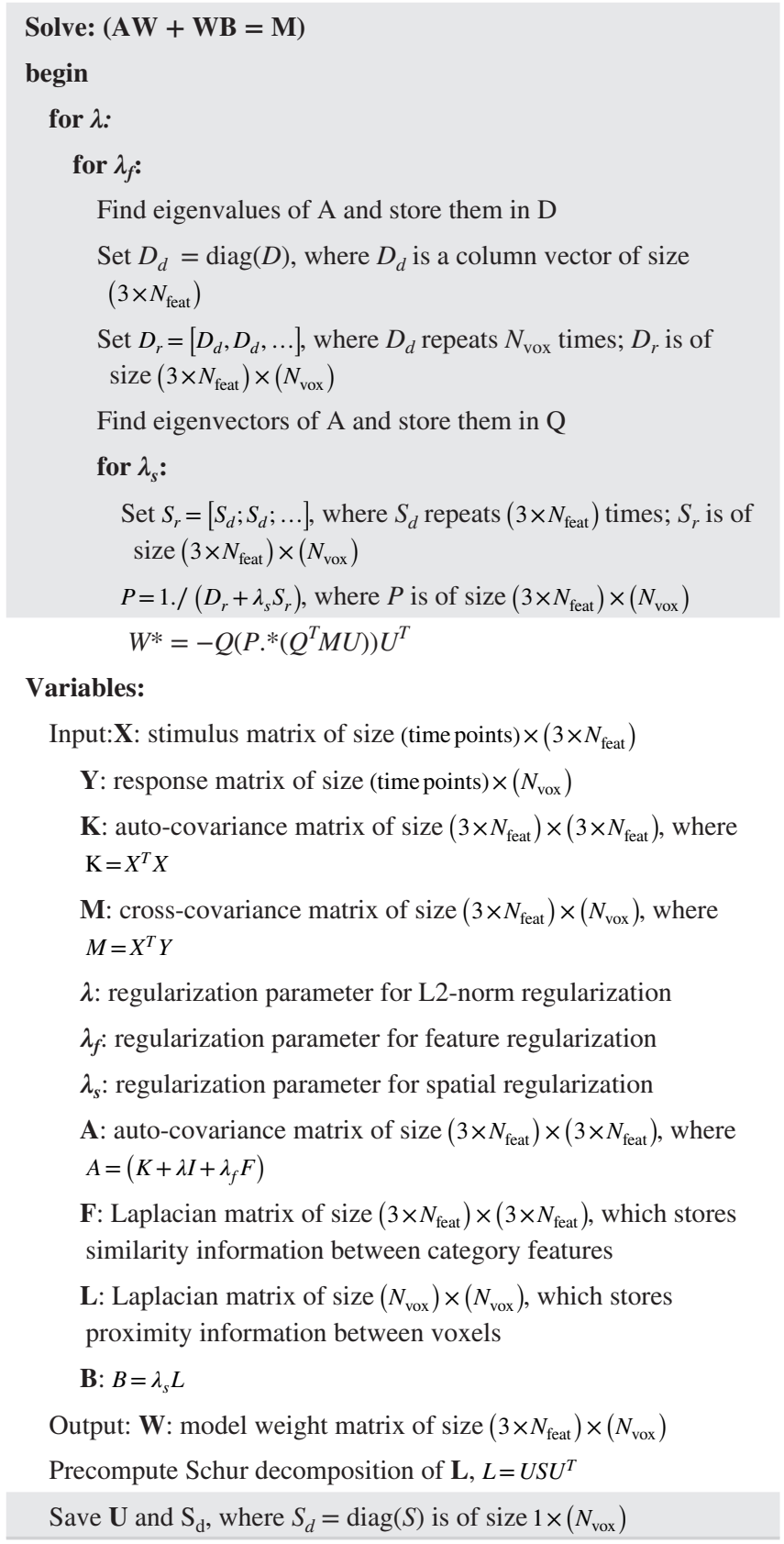




\subsection{Alternative voxelwise modeling method: graph net regularization}

As an alternative to Classical VM, voxelwise models were also fit using graph net regularization (Schoenmakers, Barth, Heskes, \& van Gerven, 2013). Graph net regularization utilizes a coupling matrix to enforce similar model weights across related features. As such, the optimization objective for Graph-Net VM is given as follows:

$$
\min _{w_{i}} \sum_{i}\left\|X w_{i}-y_{i}\right\|_{2}^{2}+\lambda\left(\sum_{i} w_{i}^{T} G w_{i}\right)
$$

Here, $G$ is a non-diagonal matrix that induces coupling between model features (Grosenick, Klingenberg, Katovich, Knutson, \& Taylor, 2013; Schoenmakers et al., 2013). $G_{j k}$ equals -1 when two features (feature- $j$ and feature- $k$ ) are coupled, 0 otherwise, and $G_{i i}$ equals the number of features that feature- $j$ is coupled to. For the category features examined in this study, $G_{j k}$ is set to -1 when the two features are semantically related (i.e., $s_{j k}>0$, see Section 2.10), 0 otherwise $\left(s_{j k}=0\right)$. Note that, unlike our proposed feature regularization method, the degree of semantic similarity is binary-valued in Graph-Net VM.

Solution to Equation 13 is computed as a single pseudo-inverse:

$$
W=\left(X^{T} X+\lambda G\right)^{\dagger} X^{T} Y
$$

Graph-Net VM was implemented via the same procedure as in Classical VM (100-fold cross-validation, the hyperparameter $\lambda$ was selected from 30 values in the range from $10^{-2}$ to $10^{7}$ ). Prediction scores were computed based on the coefficient of determination (explained variance, $R^{2}$ ) between measured and predicted BOLD responses. To measure prediction scores, a 1,000-fold jackknife resampling at a rate of $80 \%$ was used on a separate validation data (270 samples). Separately for each voxel, final prediction score was taken as the average prediction score across jackknife folds.

\subsection{Feature coherence analysis}

We hypothesized that the human brain encodes information in a way where related features elicit similar responses in single voxels (Huth et al., 2012). In the VM framework, this hypothesis would manifest itself as coherent model weights across related features for individual voxels. Because Classical VM ignores feature correlations, it can have reduced sensitivity in capturing coherence among model features. Yet, informed feature regularization enforces increased coherence among category features. Thus, in FI-VM and in JI-VM, resulting model weights are expected to be more coherent. To test this hypothesis, we defined a voxelwise feature coherence metric.
We computed the contribution of voxel- $i$ to the feature regularization cost in the regression analysis:

$$
\sigma_{\text {feature }}^{i}=\sum_{j, k} f_{j k}\left(w_{i j}-w_{i k}\right)^{2}
$$

where the differences between weights of correlated features (i.e., for pairs of [feature- $j$, feature- $k$ ] where $s_{j k}>0$, see Equation 5) are penalized according to a graph Laplacian matrix computed in the word embedding space $\left(f_{j k}\right.$ are the entries of the Laplacian matrix $F$ corresponding to feature- $j$ and feature- $k$, see Section 2.10). Voxelwise cost values were then normalized with the maximum cost obtained across the three methods (FI-, SIand JI-VM). The normalized cost was inverted to finally obtain the feature coherence metric.

\subsection{6 | Spatial coherence analysis}

Human brain encodes information across spatial clusters of neural populations (Engel, Glover, \& Wandell, 1997; Huth et al., 2012; Tootell et al., 1998). Thus, it is expected that neighboring voxels represent correlated information. As Classical VM ignores spatial correlations among neighboring voxels, it is expected to fail in capturing the coherence in neighboring voxels. Due to spatial regularization, the proposed approach should be more sensitive in capturing the spatial coherence. To test this prediction, we computed a voxelwise spatial coherence metric. The spatial coherence of voxel- $i$ was taken as the mean standard deviation among category model weights of the voxels within close spatial proximity of voxel- $i$ (i.e., for any voxel- $l$ where $c_{i l}>0$, see Section 2.11):

$$
\sigma_{\text {spatial }}^{i}=\sum_{i} c_{i l}\left\|w_{i}-w_{l}\right\|_{2}^{2}
$$

We normalized those values by dividing by the largest value computed across the three methods and then inverting the resulting value to obtain the final form of the spatial coherence metric.

\subsection{Noise ceiling}

The coefficient of determination $\left(R^{2}\right)$ between predicted response and measured response can be biased downward due to high measurement noise in voxel responses (David \& Gallant, 2005; Hsu, Borst, \& Theunissen, 2004; Sahani $\&$ Linden, 2003). As validation data were recorded a finite number of repeats (10 in this study), it is likely to contain high measurement noise in addition to signal. We calculated a noise ceiling for each voxel following procedures in Hsu et al. (Hsu et al., 2004). A voxelwise correction factor was 
calculated by dividing the maximum possible prediction score (called the noise ceiling) by the raw prediction score of each voxel. For voxels with very high level of noise, this process leads to divergent correction factors. We limited correction factors of these voxels to the average of correction factors across remaining cortical voxels.

\subsection{Statistical analysis}

Models were compared in terms of prediction score, feature coherence and spatial coherence. Specifically, changes in prediction score or coherence were calculated for various modeling methods over Classical VM. A bootstrap procedure with 10,000 samples was used to test significant changes in a given metric (prediction score or coherence) in each ROI. The null distribution was estimated via bootstrap sampling on metric values of Classical VM. The significance threshold was taken as the 95th percentile of the null distribution for each metric $(\alpha=0.05)$.

\section{3 | RESULTS}

\section{1 | Prediction scores of VM methods}

The proposed method employs additional regularization terms to consider correlations among model features as well as response correlations among voxel neighborhoods. As such, the fit models are expected to better capture voxelwise functional selectivity than Classical VM. As an alternative to Classical VM, we have also implemented Graph-Net VM (see Section 2.14, Schoenmakers et al., 2013). Yet, no significant difference was observed between Graph-Net VM and Classical VM in many regions of interest (ROIs, see Sections 2.4 and 2.5; Figure S1). Thus, subsequent comparisons were provided against Classical $\mathrm{VM}$ as the reference method. To examine the contributions of the individual regularization terms, we separately computed improvements in prediction scores of FI-VM, SI-VM and JI-VM over Classical VM (see Section 2.9 for calculation of prediction score). Figure 2 displays the change in explained variance for FI-VM, SI-VM and JI-VM in common functional ROIs. We find that FI-VM significantly improves prediction scores compared to Classical VM in all of the examined ROIs except FFA, OFA and OPA $(p<.05$, bootstrap test). The average improvement in prediction scores is $0.020 \pm 0.004\left(\Delta R^{2}\right.$, mean $\pm S D$ across ROIs) for category-selective areas (FFA, PPA, EBA, OFA and OPA), $0.017 \pm 0.003$ for attention control areas (IPS, FEF, SEF and FO) and $0.008 \pm 0.001$ for early visual areas (RET, V4). These results suggest that informed feature regularization particularly improves model accuracy in

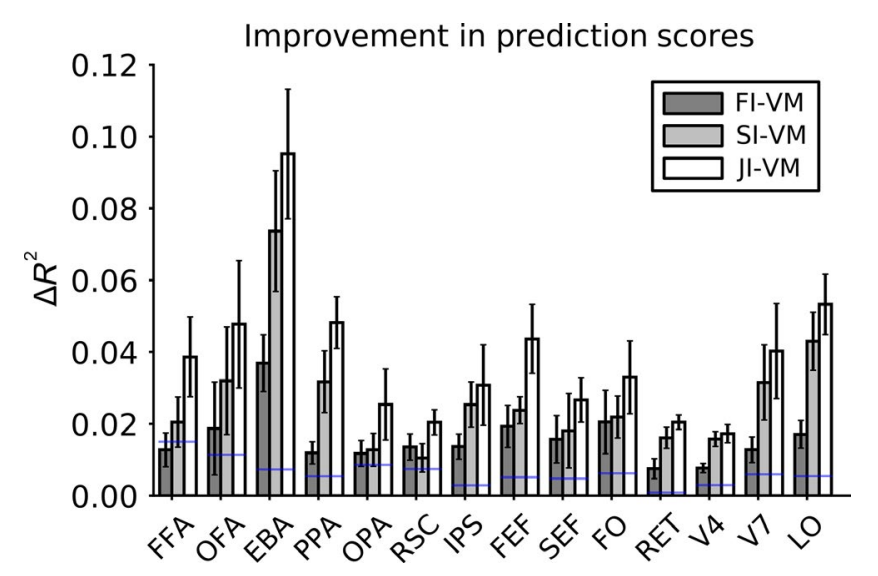

F I G URE 2 Improvement in prediction performance over VM by FI-VM, SI-VM and JI-VM. The improvement in prediction performances for the three methods (FI-, SI- and JI-VM, see Abbreviations) over VM is shown for several well-known functional ROIs (see Abbreviations). Bar graphs display improvement in prediction scores $\left(\Delta R^{2}\right.$, mean $\pm S D$ across subjects, see Section 2.9 for the definition of prediction scores). Threshold for significant improvement over Classical VM is designated with a blue line for each ROI. For FI-VM, the improvement is significant in all examined ROIs except FFA, OFA and OPA ( $p<.05$, bootstrap test). For FI-VM, improvement in prediction scores is higher in category-selective areas (FFA, PPA, EBA, OFA, OPA: $0.020 \pm 0.004$, mean $\pm S D$ across ROIs) than in attention control areas (IPS, FEF, SEF, FO: $0.017 \pm 0.003$ ) and early visual areas (RET, V4: $0.008 \pm 0.001$ ). For SI-VM, the improvement in prediction scores is significant in all ROIs except for FFA, OPA and RSC ( $p<.05$, bootstrap test). The improvement is substantial broadly across cortex $(0.039 \pm 0.007$ for category-selective areas, $0.022 \pm 0.003$ for early visual areas and $0.016 \pm 0.002$ for attention control areas). JI-VM yields significantly higher prediction scores than Classical VM in all ROIs ( $p<.05$, bootstrap test).

Improvement in prediction scores is higher in category-selective areas $(0.057 \pm 0.008)$ than in attention control areas $(0.034 \pm 0.005)$ and early visual areas $(0.019 \pm 0.002)$. These results imply that spatial and feature regularization improve model performance particularly across categoryselective areas and partly across attention control areas [Colour figure can be viewed at wileyonlinelibrary.com]

category-selective areas. These results can be attributed to the fact that representations in category-selective areas are mediated by object and action category features, whereas those in early visual areas are mediated primarily by retinotopic features (Connolly et al., 2012; Downing, Chan, Peelen, Dodds, \& Kanwisher, 2006; Engel et al., 1997; Huth et al., 2012; Naselaris et al., 2009; Op De Beeck, Haushofer, \& Kanwisher, 2008; Tootell et al., 1998).

We also find that SI-VM significantly improves prediction scores in all ROIs except for FFA, OPA and RSC $(p<.05$, bootstrap test). These improvements are substantial broadly across cortex $(0.039 \pm 0.007$ for category-selective areas, $0.022 \pm 0.003$ for attention control areas and $0.016 \pm 0.002$ for early visual areas). This result implies that spatial correlations among voxel neighborhoods exist across multiple 
stages of visual processing and use of spatial regularization improves model accuracy across these stages.

As JI-VM simultaneously leverages feature and spatial regularization, we reasoned that it should yield the highest level of improvement in explained variance over Classical VM. Indeed, we find that improvement in explained variance with JI-VM is significantly higher than Classical VM in all examined ROIs ( $p<.05$, bootstrap test). The average increase in prediction scores is $0.057 \pm 0.008$ for category-selective areas, $0.034 \pm 0.005$ for attention control areas and $0.019 \pm 0.002$ for early visual areas. In many category-selective areas, JI-VM improves prediction scores as much as the sum of individual contributions from FI-VM and SI-VM. On the other hand, in RET and V4, additional improvement by JI-VM over SI-VM seems to be limited. These results indicate that spatial and feature regularization provide independent improvements in model performance particularly across category-selective areas.

Regarding computation times, Classical VM and FI-VM lead to competing methods in terms of efficiency because both methods involve a single pseudo-inverse (see Equations 4 and 11). Two hyperparameters ( $\lambda$ and $\lambda_{f}$ ) are optimized in FI-VM, compared to a single hyperparameter in Classical $\operatorname{VM}(\lambda)$. Although calculation of the feature Laplacian matrix $(F)$ involves added burden, for a single feature set, $F$ is precomputed once and stored for later use. For our feature set (1,705 object and action categories), precomputation of the matrix $F$ took only $4 \mathrm{~min}$. The solution to SI-VM or JI-VM (see Section 2.12) then includes additional eigenvalue decompositions and matrix multiplications over a single pseudo-inverse. The complexity of the solution and the need for optimization of extra hyperparameters $\left(\lambda_{f}\right.$ and $\left.\lambda_{s}\right)$ cause a noticeable increase in computation time for SI-VM and JI-VM. The reported modeling procedures were implemented on an NVIDIA GTX 1080 card with 8 GB of VRAM, with 100fold cross-validation, 5 subjects and 30 separate values for each hyperparameter $\lambda, \lambda_{f}$ and $\lambda_{s}$. The total compute time was 3 min for Classical VM, 10 min for FI-VM, 10 min for GraphNet, $6 \mathrm{hr}$ for SI-VM and $40 \mathrm{hr}$ for JI-VM.

\section{2 | Selectivity for model features}

In the presence of high levels of noise in training data, fit models will poorly reflect the true functional selectivity of voxels. While VM uses L2-norm regularization over model features to alleviate over-fitting, high noise levels typically lead to excessive regularization that reduces sensitivity in estimating functional selectivity. In the proposed approach, we incorporate additional regularization terms across features and across voxel neighborhoods. As such, the proposed method should limit unnecessary penalization of model weights with a uniform Gaussian prior. To assess the level of weight penalization with each alternative method, in Figure 3, we plotted the optimal $\mathrm{L}_{2}$ regularization parameters over model features $(\lambda)$ on cortical flat map of a representative subject for VM, FI-VM, SI-VM and JI-VM (see Figures S2-S6 for all subjects). Among the examined methods, VM applies the highest weight penalization broadly across cortex. FI-VM reduces the degree of penalization particularly in category-selective areas, as well as in attention control areas and early visual areas. Overall, JI-VM yields the lowest level of weight penalization consistently across cortex. These results suggest that both spatial and feature regularization decrease the amount of standard L2-norm regularization over model features, thereby increasing sensitivity in measuring voxel selectivity for individual features.

We reasoned that informed feature regularization that incorporates feature correlations should enforce voxelwise models to have relatively similar weights on correlated category features and yield increased functional selectivity in individual voxels. To examine this issue, we visualized the selectivity profiles of single cortical voxels estimated with JI-VM versus VM. Figure 4 displays the selectivity profiles for JI-VM and VM for two representative voxels in EBA (extrastriate body area) and PPA (parahippocampal place area). Typically, a voxel in EBA is expected to respond selectively to categories related to "human body" while a voxel in PPA is expected to respond selectively to categories related to "places" (Downing et al., 2001; Epstein \& Kanwisher, 1998). Indeed, in voxel-1, functional selectivity for categories related to "body_parts" (e.g., "eye,"”hand," "finger" and "arm") is increased while the evoked BOLD activity by many unrelated categories is suppressed (subordinate categories of "vehicle," "artifact," etc.) in JI-VM compared to Classical VM. In voxel-2, functional selectivity for categories related to "scenes" (e.g., "road," "landscape," "street" and "path") is increased in JI-VM compared to Classical VM. Moreover, due to feature regularization, in voxel-1, model weights of the subordinate categories of "structure" (e.g., "door," "room" and "building") or "motion" (e.g., "travel" and "walk") are more similar in JI-VM compared to VM. Likewise, in voxel2 , model weights of the subordinate categories of "way" (e.g., "road," "landscape," "street" and "path") are more similar in JI-VM compared to VM. These results together indicate that informed feature regularization captures coherence in features and decreases weight penalization, and thus increases sensitivity in measuring voxelwise selectivity.

\section{3 | Feature coherence and spatial coherence of model weights}

Classical VM ignores feature correlations, so it has reduced sensitivity to capture coherence in model features. FI-VM and JI-VM, on the other hand, employ regularization that 

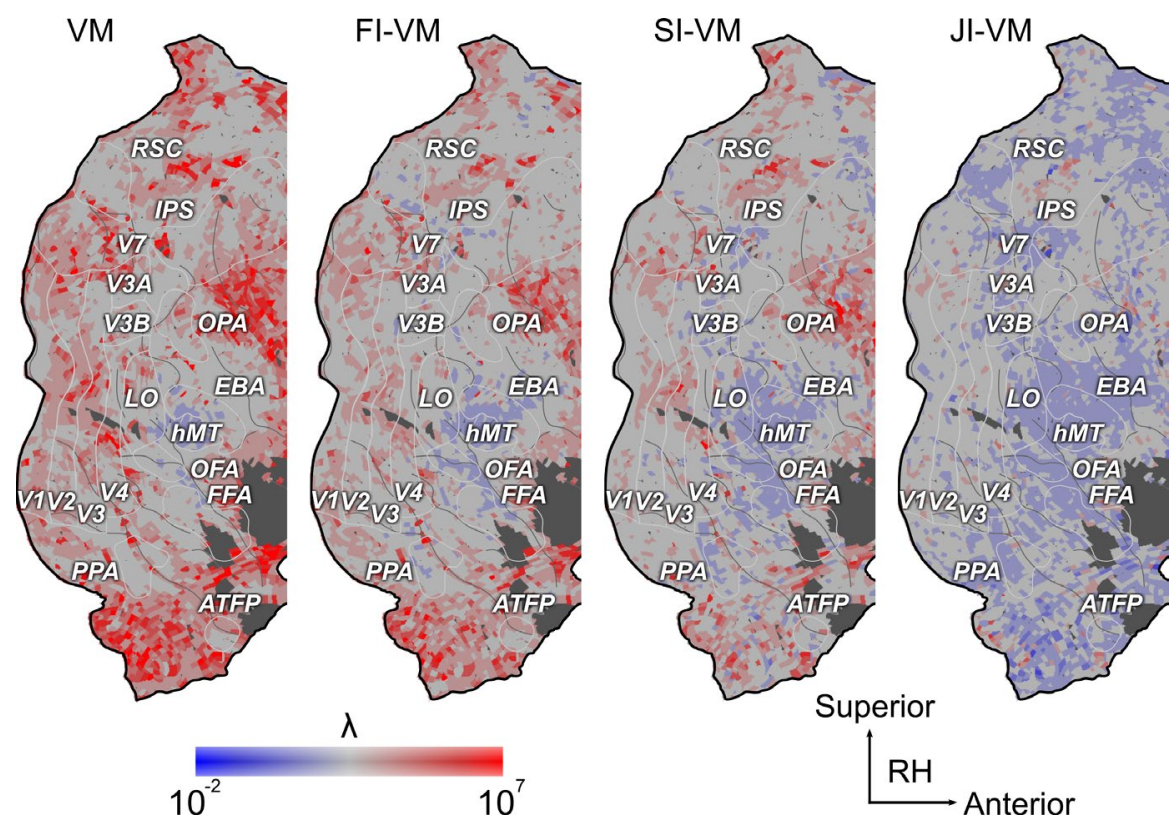

F I G URE 3 Cortical distribution of optimal L2-norm regularization parameter. The $\mathrm{L}_{2}$ regularization parameters over model features in the four VM methods (VM, FI-VM, SI-VM and JI-VM) are visualized on the right hemisphere of subject S1 (see Abbreviations). VM strictly penalizes model weights broadly across cortex. High regularization parameters reduce sensitivity in measuring voxelwise functional selectivity. Here, we incorporate additional regularization terms across features and across voxel neighborhoods. As such, the proposed method should limit unnecessary penalization of model weights by $\mathrm{L}_{2}$ regularization. As a result, compared to VM, FI-VM applies less weight regularization particularly in categoryselective areas, early visual areas and frontal gyrus. Overall, JI-VM enforces the lowest weight penalization among the competing methods consistently across cortex. These results imply that both spatial and feature regularization alleviate the need for $\mathrm{L}_{2}$ regularization, thereby increasing sensitivity in measuring voxelwise functional selectivity [Colour figure can be viewed at wileyonlinelibrary.com]

respects feature correlations, so they should better capture feature coherence. To test this prediction, we computed a voxelwise feature coherence metric and visualized it on flat maps (see Section 2.15). In Figure 5, feature coherence of voxelwise models is shown on the posterior part of the cortex of a representative subject (see Figures S7-S11 for all subjects). Feature coherence of FI-VM and JI-VM models is significantly higher than VM and SI-VM in all ROIs ( $p<.05$, bootstrap test). In attention control areas, the increase in feature coherence is very high (see Figure 6; $14.11 \pm 1.59 \%$ for FI-VM and $20.45 \pm 2.41 \%$ for JI-VM). In category-selective areas, the increase in feature coherence is substantial (see Figure $6 ; 8.66 \pm 1.35 \%$ for FI-VM and $14.33 \pm 1.69 \%$ for JI-VM). In early visual areas, the increase is relatively modest $(4.47 \pm 0.67 \%$ for FI-VM and $6.65 \pm 0.92 \%$ for JI-VM $)$. These results suggest that informed feature regularization increases feature coherence particularly in attention control areas and high-level visual areas.

F I G URE 4 Functional selectivity in a single voxel. Functional selectivity of two well-modeled voxels in EBA (extrastriate body area, voxel-1, S1) and PPA (parahippocampal place area, voxel-2, S2) is visualized for several object and action categories organized under the WordNet hierarchy. Each node represents the estimated response of the voxel to the labeled object (circles) or action category (squares). Red nodes correspond to categories that evoke above-mean responses, and blue nodes correspond to categories that evoke below-mean responses. Sizes of the nodes indicate the magnitude of the evoked responses. For visualization purposes, the model weights are normalized within each voxel. In the proposed approach, we incorporate feature and spatial regularization that limit unnecessary penalization of model weights by $\mathrm{L}_{2}$ regularization while improving prediction scores $\left(R^{2}=.677\right.$ vs. $R^{2}=.839$ for voxel- 1 and $R^{2}=.029 \mathrm{vs} . \mathrm{R}^{2}=.105$ for voxel-2). Typically, a voxel in EBA is expected to respond selectively to categories related to "human body" while a voxel in PPA is expected to respond selectively to categories related to "places." Indeed, in voxel-1, functional selectivity for categories related to "body_parts" (e.g., "eye," "hand," "finger" and "arm") is increased while the evoked BOLD activity by many unrelated categories is suppressed (subordinate categories of "vehicle," "artifact," etc.) in JI-VM compared to Classical VM. In voxel-2, functional selectivity for categories related to "scenes" (e.g., "road," "landscape," "street" and "path") is increased. Furthermore, feature regularization enforces more similar weights in correlated categories. For example, in voxel-1, model weights of the subordinate categories of "structure" (e.g., "door," "room" and "building") or "motion" (e.g., "travel" and "walk") are more similar in JI-VM compared to VM. Likewise, in voxel-2, model weights of the subordinate categories of "way" (e.g., "road," "landscape," "street" and "path") are more similar in JI-VM compared to VM. Taken together, these results imply that feature and spatial regularization increase sensitivity in assessment of functional selectivity by enforcing functionally coherent model weights [Colour figure can be viewed at wileyonlinelibrary.com] 
Spatial regularization enforces spatially coherent model weights for neighboring voxels, so SI-VM and JI-VM should yield higher spatial coherence compared to the alternative methods. To test this prediction, we computed a voxelwise spatial coherence metric and visualized it on flat maps (see Section 2.16). In Figure 7, spatial coherence of voxelwise models on the posterior part of the cortex of a representative subject is shown (see Figures S12-S16 for all subjects). SI-VM significantly increases and FI-VM significantly decreases spatial coherence consistently in all ROIs ( $p<.05$, bootstrap test). Meanwhile, JI-VM has significantly increased spatial coherence in all ROIs except RSC, FEF and FO ( $p<.05$, bootstrap test). Yet, JI-VM shows significantly decreased spatial coherence in SEF. For JI$\mathrm{VM}$, the level of increase in coherence is higher in category-selective areas $(10.19 \pm 1.59 \%)$ than in attention control areas $(2.38 \pm 2.91 \%)$ and early visual areas $(3.06 \pm 0.85 \%$; Figure 8$)$. These results imply that spatial regularization yields improved spatial coherence broadly across cortex.

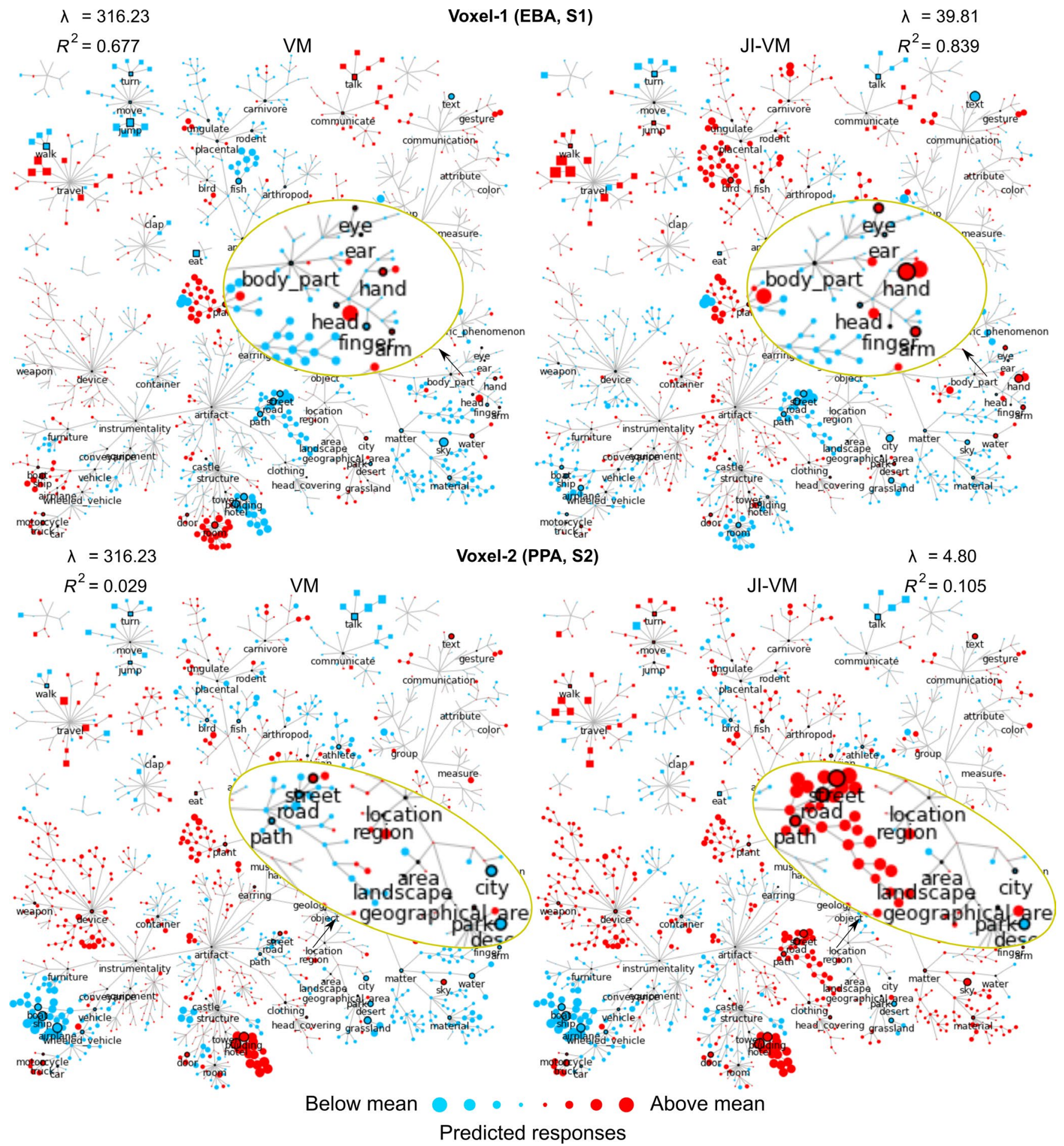




\section{4 | DISCUSSION}

Voxelwise modeling (VM) is a powerful tool to model single-voxel selectivity for natural stimulus features with high sensitivity (Kay et al., 2008; Naselaris et al., 2009). Still, VM can yield suboptimal performance in the presence of high levels of measurement noise. Classical VM does not consider potential correlations among model features and spatial correlations among neighboring voxels. To improve performance, here we first introduced an informed feature regularization that considers feature correlations. We also introduced a spatial regularization approach that constructs voxel neighborhoods based on cortical distances. Our results indicate that the proposed approach provides more predictive voxelwise models for a natural vision experiment in many regions across cortex. We further measured the coherence of fit models, and our results suggest that improvements in prediction performance can be attributed to increased spatial and feature coherence across cortical representations.

Voxelwise modeling aims to estimate single-voxel functional selectivity from stimulus-driven fMRI data. Yet, VM may show suboptimal sensitivity in measuring selectivity in the presence of high noise levels and for complex stimulus models with thousands of features. Here, two sources of prior information were examined to alleviate this limitation, namely correlations among stimulus features and correlations among voxel responses. These priors were incorporated to the VM framework as added feature and spatial regularization terms (both in JI-VM, feature reg. in FI-VM and spatial reg. in SI-VM). Note that regularized problem solutions require a careful trade-off between information from collected data (stimulus-response data) and prior knowledge. As FI-VM is constrained to only leverage feature regularization, it may alleviate noise at the expense of excessively increased feature coherence. Likewise, SI-VM can alleviate noise at the expense of over-increase in spatial coherence. (A similar problem is observed in traditional $\mathrm{VM}$ with excessive $\mathrm{L}_{2}$ regularization, Figure 3.) In contrast, JI-VM has increased diversity in the type of prior information it can leverage. The success of a model in measuring functional selectivity should be primarily judged by the prediction scores. Thus, higher prediction scores of JI-VM compared to competing methods suggest that it can maintain a better balance between feature and spatial coherence while alleviating noise. However, it should also be noted that higher prediction performances of SI-VM and JI-VM over FI-VM come at the expense of increased computation time. Trade-off between high prediction performance and low computation time can be an important criterion in deciding which method to use.

With similar motivations to treat feature correlations, a recent study calculated a linear transformation of a category model to impose regularization with a non-uniform prior on model features (Nunez-Elizalde et al., 2018). The transformation was defined based on the dimensions of a word
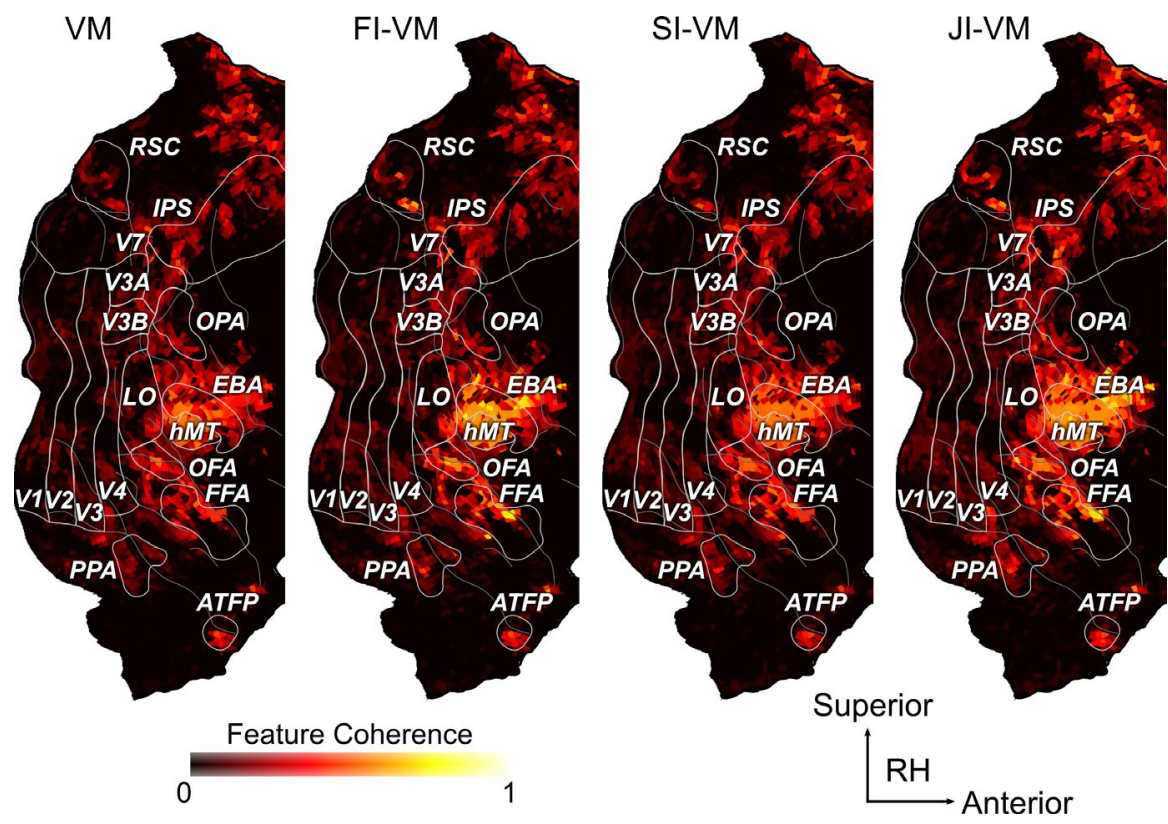

F I G U RE 5 Feature coherence for VM, FI-VM, SI-VM and JI-VM. Feature coherence for subject S1 is shown on the posterior part of the right hemisphere (see Abbreviations). VM ignores potential feature correlations in the stimulus, so it has reduced sensitivity to coherence in stimulus features. Informed feature regularization enforces similar model weights for correlated model features. Therefore, feature coherence of FI-VM and JI-VM is higher than that of VM and SI-VM across many cortical regions including category-selective areas, attention control areas and early visual areas. These results indicate that informed feature regularization better captures feature coherence broadly across visual cortex [Colour figure can be viewed at wileyonlinelibrary.com] 


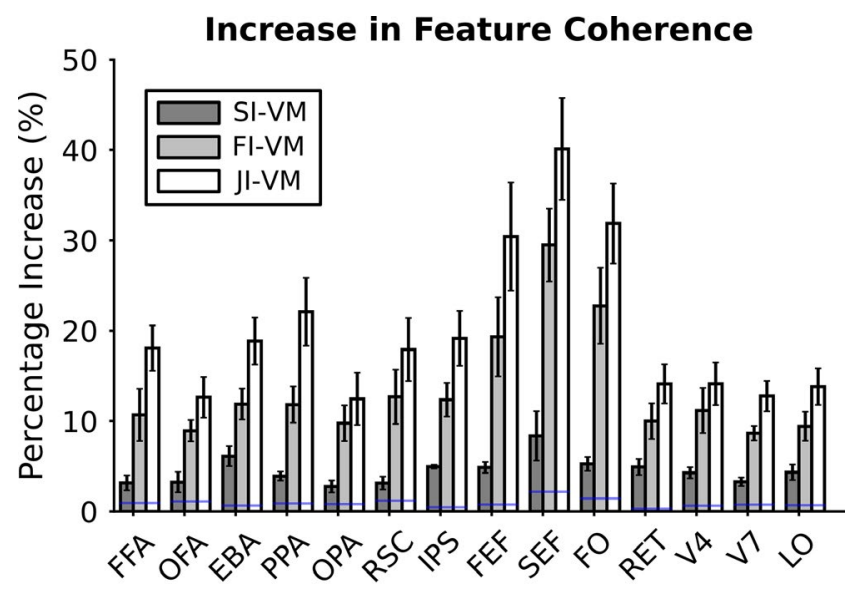

FIGURE 6 Feature coherence across functional ROIs. Bar graphs display increases in feature coherence by the three methods (SI-VM, FI$\mathrm{VM}$ and JI-VM) over VM (mean $\pm S D$ across subjects). Feature coherence of FI-VM and JI-VM models is significantly higher than that of VM in all functional ROIs ( $p<.05$, bootstrap test). Threshold for significant improvement over Classical VM is designated with a blue line for each ROI. The average increase in attention control areas is $14.11 \pm 1.59 \%$ for FI-VM and $20.45 \pm 2.41 \%$ for JI-VM, whereas the average increase in category-selective areas is $8.66 \pm 1.35 \%$ for FI-VM and $14.33 \pm 1.69 \%$ for $\mathrm{JI}-\mathrm{VM}$ and the average increase in early visual areas is $4.47 \pm 0.67 \%$ for FI-VM and $6.65 \pm 0.92 \%$ for JI-VM. In contrast, SI-VM that lacks feature regularization has relatively limited increase in feature coherence across all ROIs. These results suggest that informed feature regularization increases functional coherence in cortical representations particularly in areas that take part in attention control and high-level visual functions [Colour figure can be viewed at wileyonlinelibrary.com]

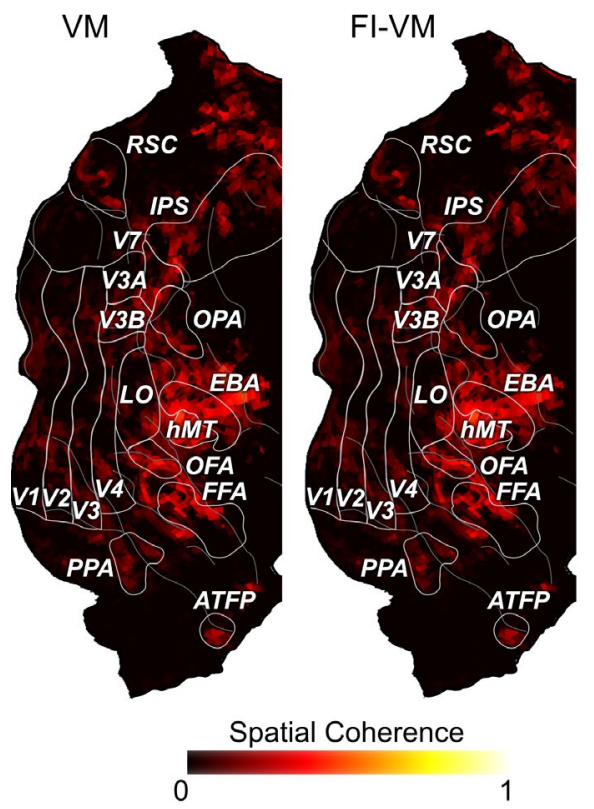

embedding space constructed using co-occurrence statistics in a large text corpus. It was reported that the proposed transformation approach yielded improved prediction scores for category models across significantly predicted cortical voxels. As opposed to a transformation of model features, here we leave the model features in their original space and leverage potential correlations through a graph Laplacian-based regularization term. Avoiding an additional transformation might offer an advantage in terms of interpretability of resulting model weights. Our approach also allows for independent weighing of differences of model weights for each pair of category features. It remains important future work to comparatively demonstrate the transformation versus regularization approach for treatment of feature correlations.

Regularization across model features has also been leveraged in neuroimaging studies beyond fMRI (Tran, Phung, Luo, \& Venkatesh, 2015; Zhu, Suk, Wang, Lee, \& Shen, 2015) or studies that focus on modeling natural stimuli (Sandler, Blitzer, Pratim Talukdar, \& Ungar, 2008; Zhang \& Ostendorf, 2012). A typical way that feature correlations are leveraged is to reduce dimensionality by eliminating redundant features from subsequent analyses. A previous study incorporated feature regularization to improve AD diagnosis performance with both regression and classification models (Zhu et al., 2015). The authors estimated similarity of neuroimaging features on MRI and PET images, and then used these estimates to regularize correlated features during modeling. They have reported increased

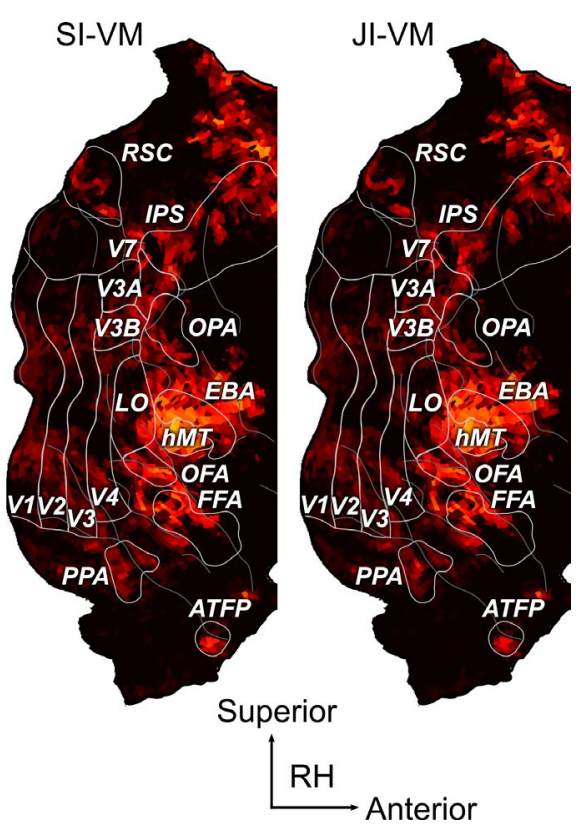

FIGURE 7 Spatial coherence for VM, FI-VM, SI-VM and JI-VM. Spatial coherence for subject S1 is shown on the posterior part of the right hemisphere (see Abbreviations). VM ignores correlations among voxel neighborhoods, so it has reduced sensitivity to spatial coherence in cortical representations. Spatial regularization enforces increased spatial coherence among cortical representations within voxel neighborhoods. Although FI-VM and VM have relatively similar spatial coherence values, SI-VM and JI-VM yield higher spatial coherence consistently across many cortical regions. These results suggest that spatial regularization better captures spatial coherence broadly across visual cortex [Colour figure can be viewed at wileyonlinelibrary.com] 


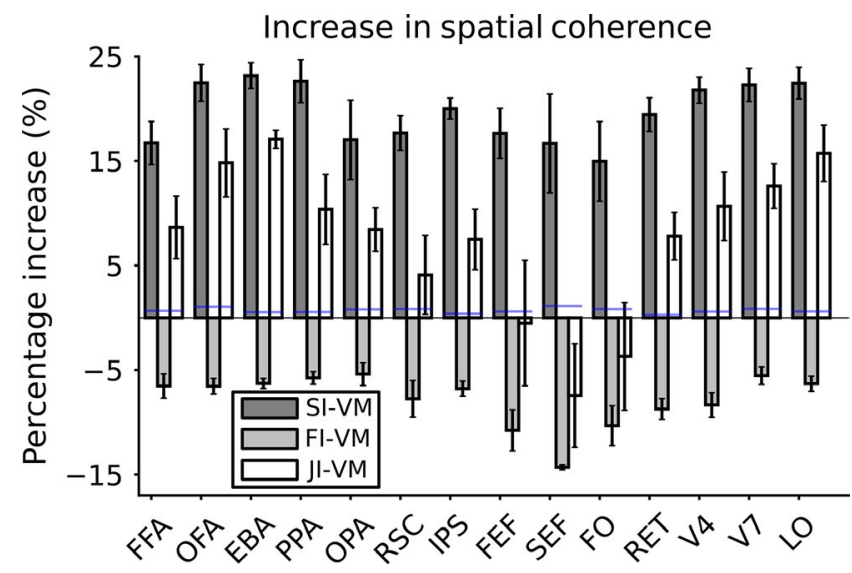

F IG U RE 8 Spatial coherence across functional ROIs. Bar graphs display increases in spatial coherence by the three methods (FI-VM, SIVM and JI-VM) over VM (mean $\pm S D$ across subjects, see Abbreviations). Spatial regularization enforces increased spatial coherence among cortical representations of neighboring voxels. Threshold for significant improvement over Classical VM is designated with a blue line for each ROI. SI-VM has significantly higher spatial coherence than VM in all functional ROIs ( $p<.05$, bootstrap test). JI-VM has significantly higher spatial coherence than VM in all functional ROIs except RSC, FEF, SEF and FO $(p<.05$, bootstrap test). The average increase in category-selective areas is $17.00 \pm 0.69 \%$ for SI-VM and $10.19 \pm 1.59 \%$ for JI-VM, whereas the average increase in attention control areas is $14.89 \pm 1.29 \%$ for SI-VM and $2.38 \pm 2.91 \%$ for JI-VM and the average increase in early visual areas is $7.89 \pm 0.22 \%$ for SI-VM and $3.06 \pm 0.85 \%$ for JI-VM. Meanwhile, FI-VM that lacks spatial regularization does not yield any improvement in spatial coherence. These results indicate that spatial regularization increases spatial coherence among cortical representations of voxel neighborhoods across many cortical regions [Colour figure can be viewed at wileyonlinelibrary.com]

regression and classification performance in AD diagnosis. In another study on medical risk stratification, the authors implemented feature regularization on various features extracted from electronic medical records (EMRs) on different timescales during ordinal regression (Tran et al., 2015). They have reported that suicide risk can be better predicted by a model of EMR-derived features compared to clinicians. In both studies, the authors discarded redundant features via an L1-norm regularization. Unlike these previous studies, here we retained all stimulus features in the model. Several previous studies have employed a feature regularization approach similar to the one proposed here for robust text classification (Sandler et al., 2008; Zhang \& Ostendorf, 2012). In Zhang et al., the authors regularized the correlated subtopics for a discriminative classifier. They constructed a feature affinity matrix using subtopic co-occurrences on a large unlabeled dataset as in our method. Later, they included a feature regularization term based on the feature affinity matrix into maximum entropy training objective. They have reported that feature regularization on subtopic features improved text classification accuracy over other semi-supervised learning approaches. In Sandler et al., the authors incorporated feature regularization into sentiment classification and newsgroups document classification via logistic regression. Similar to our approach, they used a graph Laplacian-based regularization by using both word co-occurrences and prior domain information to compute feature similarities. They have reported improved classification performances over many other semi-supervised learning methods.

The proposed method leverages a linguistic model to guide feature regularization on a visual category model. Indeed, several recent studies suggest that category information in natural visual scenes is well captured by semantic features in natural language. Clarke et al. reported that visual objects that shared a superordinate category yielded more similar activation patterns in perirhinal cortex than those that did not (Clarke \& Tyler, 2015). Carlson et al. reported that semantically related objects had similar neural representations in inferior temporal cortex (Carlson, Simmons, Kriegeskorte, $\&$ Slevc, 2014). Semantic relatedness was measured using hierarchical word relations and distributional patterns of words in large text corpora. More recently, Wen et al. reported a significant correlation between representational similarity and semantic similarity across cortex for a pair of visual objects (Wen, Shi, Chen, \& Liu, 2018). In that study, semantic similarity was measured based on the distances in word embedding models (Landauer, 2006; Pennington, Socher, \& Manning, 2014).

Here, we used a common set of hyperparameters for constructing the feature Laplacian matrix and spatial Laplacian matrix. A potential avenue for improvement for the proposed method is to consider voxelwise optimization of these parameters. Note, however, that this will require considerably higher computational load. Improving performance in feature regularization might also be viable via different approaches to computing similarity of model features. Here, we calculated them in the word embedding space constructed using co-occurrence statistics. One can typically utilize another word embedding space constructed by word2vec or LSA to find the similarities of model features (Landauer, 2006; Mikolov, Yih, \& Zweig, 2013).

In summary, we introduced a novel voxelwise modeling approach that simultaneously utilizes stimulus correlations in model features and response correlations among voxel neighborhoods. Our results indicate clear improvements in prediction performance, spatial coherence and feature coherence of category models in a natural vision experiment, specifically across visual category areas and attention control areas. With little increase in computation time, a significant albeit modest improvement in prediction performance is obtained with feature regularization compared to spatial regularization. Meanwhile, concurrent use of spatial and feature regularization yields even higher prediction performance with further 
increase in computation load. These improvements are best attributed to prevention of crude, unnecessary penalization of model weights, and thereby increased accuracy in measurement of functional selectivity. While demonstrations were primarily for a category model, the proposed approach can also be beneficial to modeling of other types of stimulus features known to exhibit correlations. Overall, the proposed approach can offer improved sensitivity in modeling of single voxels in naturalistic fMRI experiments.

\section{ACKNOWLEDGEMENTS}

We thank A. Vu, N. Bilenko, J. Gao, A. Huth, S. Nishimoto and J.L. Gallant for assistance in various aspects of this research. This work was supported in part by a National Eye Institute Grant (grant number EY019684), by a Marie Curie Action Career Integration Grant (grant number PCIG13-GA-2013-618101), by a European Molecular Biology Organization Installation Grant (grant number IG 3028), by a TUBA GEBIP 2015 fellowship, by a BAGEP 2017 fellowship, by an ASELSAN PhD scholarship, by a TUBITAK-BIDEB 2211 scholarship and by an NVIDIA GPU Grant.

\section{CONFLICTS OF INTEREST}

No conflicts of interest, financial or otherwise, are declared by the authors.

\section{AUTHORCONTRIBUTIONS}

Ö.Y., E.Ç. and T.Ç. conceived and designed the research; Ö.Y., E.Ç. and T.Ç. performed the experiments; Ö.Y. and T.Ç. analyzed the data; Ö.Y., E.Ç. and T.Ç. interpreted the results of the experiments; Ö.Y. prepared the figures; Ö.Y. and T.Ç. drafted, edited and revised the manuscript; Ö.Y., E.Ç. and T.Ç. approved the final version of the manuscript.

\section{DATA AVAILABILITY STATEMENT}

Code and dataset used in the current study are available from the corresponding author on reasonable request: Please contact cukur@ee.bilkent.edu.tr.

\section{ORCID}

Özgür Yllmaz (DD https://orcid.org/0000-0001-7375-9171

Tolga Çukur (D) https://orcid.org/0000-0002-2296-851X

\section{REFERENCES}

Bishop, C. M. (2013). Pattern recognition and machine learning. Delhi, NY: Springer.

Carlson, T. A., Simmons, R. A., Kriegeskorte, N., \& Slevc, L. R. (2014). The emergence of semantic meaning in the ventral temporal pathway. Journal of Cognitive Neuroscience, 26, 120-131.

Çelik, E., Dar, S. U. H., Yılmaz, Ö., Keleş, Ü., \& Çukur, T. (2019). Spatially informed voxelwise modeling for naturalistic fMRI experiments. NeuroImage, 186, 741-757. https://doi.org/10.1016/j.neuro image.2018.11.044
Clarke, A., \& Tyler, L. K. (2015). Understanding what we see: How we derive meaning from vision. Trends in Cognitive Sciences, 19, $677-687$.

Connolly, A. C., Guntupalli, J. S., Gors, J., Hanke, M., Halchenko, Y. O., Wu, Y.-C., ... Haxby, J. V. (2012). The representation of biological classes in the human brain. Journal of Neuroscience, 32(8), 2608-2618. https://doi.org/10.1523/JNEUROSCI.5547-11.2012

Çukur, T., Huth, A. G., Nishimoto, S., \& Gallant, J. L. (2013). Functional subdomains within human FFA. Journal of Neuroscience, 33, 16748-16766.

Çukur, T., Nishimoto, S., Huth, A. G., \& Gallant, J. L. (2013). Attention during natural vision warps semantic representation across the human brain. Nature Neuroscience, 16, 763-770.

Dale, A. M., Fischl, B., \& Sereno, M. I. (1999). Cortical surface-based analysis: I. Segmentation and surface reconstruction. NeuroImage, 9, 179-194. https://doi.org/10.1006/nimg.1998.0395

David, S. V., \& Gallant, J. L. (2005). Predicting neuronal responses during natural vision. Network: Computation in Neural Systems, 16, 239-260

Deerwester, S., Dumais, S. T., Furnas, G. W., Landauer, T. K., \& Harshman, R. (1990). Indexing by latent semantic analysis. Journal of the American Society for Information Science and Technology, 41, 391-407.

Downing, P. E., Chan, A.-W.-Y., Peelen, M. V., Dodds, C. M., \& Kanwisher, N. (2006). Domain specificity in visual cortex. Cerebral Cortex, 16, 1453-1461. https://doi.org/10.1093/cercor/bhj086

Downing, P. E., Jiang, Y., Shuman, M., \& Kanwisher, N. (2001). A cortical area selective for visual processing of the human body. Science, 293, 2470-2473.

Engel, S. A., Glover, G. H., \& Wandell, B. A. (1997). Retinotopic organization in human visual cortex and the spatial precision of functional MRI. Cerebral Cortex, 7, 181-192. https://doi.org/10.1093/ cercor/7.2.181

Epstein, R., \& Kanwisher, N. (1998). A cortical representation of the local visual environment. Nature, 392, 598-601. https://doi. org/10.1038/33402

Erwin, E., Obermayer, K., \& Schulten, K. (1995). Models of orientation and ocular dominance columns in the visual cortex: A critical comparison. Neural Computation, 7, 425-468. https://doi.org/10.1162/ neco.1995.7.3.425

Gao, J. S., Huth, A. G., Lescroart, M. D., \& Gallant, J. L. (2015). Pycortex: An interactive surface visualizer for fMRI. Frontiers in Neuroinformatics, 9, 23.

Grosenick, L., Klingenberg, B., Katovich, K., Knutson, B., \& Taylor, J. E. (2013). Interpretable whole-brain prediction analysis with GraphNet. NeuroImage, 72, 304-321. https://doi.org/10.1016/j. neuroimage.2012.12.062

Hoerl, A. E., \& Kennard, R. W. (1970). Ridge Regression: Biased estimation for nonorthogonal problems. Technometrics, 12, 55-67. https://doi.org/10.1080/00401706.1970.10488634

Hsu, A., Borst, A., \& Theunissen, F. E. (2004). Quantifying variability in neural responses and its application for the validation of model predictions. Network, 15(2), 91-109. https://doi. org/10.1088/0954-898X_15_2_002

Huth, A. G., deHeer, W. A., Griffiths, T. L., Theunissen, F. E., \& Gallant, J. L. (2016). Natural speech reveals the semantic maps that tile human cerebral cortex. Nature, 532, 453-458. https://doi. org/10.1038/nature17637

Huth, A. G., Nishimoto, S., Vu, A. T., \& Gallant, J. L. (2012). A continuous semantic space describes the representation of thousands of 
object and action categories across the human brain. Neuron, 76, 1210-1224. https://doi.org/10.1016/j.neuron.2012.10.014

Kanwisher, N., McDermott, J., \& Chun, M. M. (1997). The fusiform face area: A module in human extrastriate cortex specialized for face perception. Journal of Neuroscience, 17, 4302-4311. https:// doi.org/10.1523/NEUROSCI.17-11-04302.1997

Kay, K. N., Naselaris, T., Prenger, R. J., \& Gallant, J. L. (2008). Identifying natural images from human brain activity. Nature, 452, 352-355. https://doi.org/10.1038/nature06713

Landauer, T. K. (2006). Latent semantic analysis. In L. Nadel (Ed.), Encyclopedia of cognitive science. Chichester, UK: John Wiley \& Sons, Ltd.

Lescroart, M. D., Stansbury, D. E., \& Gallant, J. L. (2015). Fourier power, subjective distance, and object categories all provide plausible models of BOLD responses in scene-selective visual areas. Frontiers in Computational Neuroscience, 9, 135.

Lund, K., \& Burgess, C. (1996). Producing high-dimensional semantic spaces from lexical co-occurrence. Behavior Research Methods, Instruments, \& Computers, 28, 203-208.

Mikolov, T., Yih, W., \& Zweig, G. (2013). Linguistic regularities in continuous space word representations. In Proceedings of the 2013 Conference of the North American Chapter of the Association for Computational Linguistics: Human language technologies (pp. 746-751).

Miller, G. A., \& Miller, G. A. (1995). WordNet: A lexical database for English. Communications of the ACM, 38, 39-41. https://doi. org/10.1145/219717.219748

Mitchell, T. M., Shinkareva, S. V., Carlson, A., Chang, K.-M., Malave, V. L., Mason, R. A., \& Just, M. A. (2008). Predicting human brain activity associated with the meanings of nouns. Science, 320, 1191-1195.

Nakamura, K. (2000). Functional delineation of the human occipito-temporal areas related to face and scene processing: A PET study. Brain, 123, 1903-1912. https://doi.org/10.1093/brain /123.9.1903

Naselaris, T., Prenger, R. J., Kay, K. N., Oliver, M., \& Gallant, J. L. (2009). Bayesian reconstruction of natural images from human brain activity. Neuron, 63, 902-915. https://doi.org/10.1016/j. neuron.2009.09.006

Nishimoto, S., Vu, A. T., Naselaris, T., Benjamini, Y., Yu, B., \& Gallant, J. L. (2011). Reconstructing visual experiences from brain activity evoked by natural movies. Current Biology, 21, 1641-1646.

Nunez-Elizalde, A. O., Huth, A. G., \& Gallant, J. L. (2018). Voxelwise encoding models with non-spherical multivariate normal priors. bioRxiv, 386318.

Op De Beeck, H. P., Haushofer, J., \& Kanwisher, N. G. (2008). Interpreting fMRI data: Maps, modules and dimensions. Nature Reviews Neuroscience, 9(2), 123-135. https://doi.org/10.1038/nrn2314

Pennington, J., Socher, R., \& Manning, C. (2014). Glove: Global vectors for word representation. In Proceedings of the 2014 conference on empirical methods in natural language processing (EMNLP) (pp. 1532-1543). Association for Computational Linguistics, Stroudsburg, PA, USA.

Sahani, M., \& Linden, J. F. (2003). How Linear are Auditory Cortical Responses?. Advances in Neural Information Processing Systems, $15,125-132$.

Sandler, T., Blitzer, J., Pratim Talukdar, P., \& Ungar, L. (2008). Regularized Learning with Networks of Features. Advances in Neural Information Processing Systems 21 - Proceedings of the 2008 Conference.(-).
Schoenmakers, S., Barth, M., Heskes, T., \& vanGerven, M. (2013). Linear reconstruction of perceived images from human brain activity. NeuroImage, 83, 951-961. https://doi.org/10.1016/j.neuro image.2013.07.043

Tootell, R. B., Hadjikhani, N. K., Vanduffel, W., Liu, A. K., Mendola, J. D., Sereno, M. I., \& Dale, A. M. (1998). Functional analysis of primary visual cortex (V1) in humans. Proceedings of the National Academy of Sciences of the United States of America, 95, 811-817.

Tran, T., Phung, D., Luo, W., \& Venkatesh, S. (2015). Stabilized sparse ordinal regression for medical risk stratification. Knowledge and Information Systems, 43, 555-582.

Tucholka, A., Fritsch, V., Poline, J.-B., \& Thirion, B. (2012). An empirical comparison of surface-based and volume-based group studies in neuroimaging. NeuroImage, 63, 1443-1453. https://doi. org/10.1016/j.neuroimage.2012.06.019

Turney, P. D., \& Pantel, P. (2010). From frequency to meaning: Vector space models of semantics. Journal of Artificial Intelligence Research, 37, 141-188.

Van Essen, D. C., Drury, H. A., Joshi, S., \& Miller, M. I. (1998). Functional and structural mapping of human cerebral cortex: Solutions are in the surfaces. Proceedings of the National Academy of Sciences of the United States of America, 95, 788-795.

Wehbe, L., Murphy, B., Talukdar, P., Fyshe, A., Ramdas, A., \& Mitchell, T. (2014). Simultaneously uncovering the patterns of brain regions involved in different story reading subprocesses. PLOS ONE, 9, e112575. https://doi.org/10.1371/journ al.pone.0112575

Wen, H., Shi, J., Chen, W., \& Liu, Z. (2018). Deep residual network predicts cortical representation and organization of visual features for rapid categorization. Scientific Reports, 8, 3752.

Woolrich, M. W., Jbabdi, S., Patenaude, B., Chappell, M., Makni, S., Behrens, T., ... Smith, S. M. (2009). Bayesian analysis of neuroimaging data in FSL. NeuroImage, 45, S173-S186. https://doi. org/10.1016/j.neuroimage.2008.10.055

Zarahn, E., Aguirre, G. K., \& D’Esposito, M. (1997). Empirical analyses of BOLD fMRI statistics. I. Spatially unsmoothed data collected under null-hypothesis conditions. NeuroImage, 5, 179-197. https:// doi.org/10.1006/nimg.1997.0263

Zhang, B., \& Ostendorf, M. (2012). Semi-supervised learning for text classification using feature affinity regularization. MLSLP.

Zhu, X., Suk, H.-I., Wang, L., Lee, S.-W., \& Shen, D. (2017). A novel relational regularization feature selection method for joint regression and classification in AD diagnosis. Medical Image Analysis, 38, 205-214. https://doi.org/10.1016/j.media.2015.10.008

\section{SUPPORTING INFORMATION}

Additional supporting information may be found online in the Supporting Information section.

How to cite this article: Yılmaz Ö, Çelik E, Çukur T. Informed feature regularization in voxelwise modeling for naturalistic fMRI experiments. Eur J Neurosci. 2020;52:3394-3410. https://doi.org/10.1111/ejn.14760 